“ (C) 2017 IEEE. Personal use of this material is permitted. Permission from IEEE must be obtained for all other uses, in any current or future media, including

reprinting/republishing this material for advertising or promotional purposes, creating new collective works, for resale or redistribution to servers or lists, or reuse of any copyrighted component of this work in other works." 


\title{
The Need of an Optimal QoS Repository and Assessment Framework in Forming a Trusted Relationship in Cloud
}

\author{
A Systematic Review \\ Ahmed Alghamdi*, Walayat Hussain ${ }^{*}$, Abdulaziz Alharthi ${ }^{*}$, Abdullah Badar Almusheqah ${ }^{*}$ \\ Faculty of Engineering and IT, University of Technology Sydney Australia \\ +Walayat.hussain]@uts.edu.au, “[abdulaziz.n.alharthi, ahmed.alghamdi, \\ abdullahbadera.almusheqah]@student.uts.edu.au
}

\begin{abstract}
Due to the cost-effectiveness and scalable features of the cloud the demand of its services is increasing every next day. Quality of Service (QOS) is one of the crucial factor in forming a viable Service Level Agreement (SLA) between a consumer and the provider that enable them to establish and maintain a trusted relationship with each other. SLA identifies and depicts the service requirements of the user and the level of service promised by provider. Availability of enormous service solutions is troublesome for cloud users in selecting the right service provider both in terms of price and the degree of promised services. On the other end a service provider need a centralized and reliable QoS repository and assessment framework that help them in offering an optimal amount of marginal resources to requested consumer. Although there are number of existing literatures that assist the interaction parties to achieve their desired goal in some way, however, there are still many gaps that need to be filled for establishing and maintaining a trusted relationship between them. In this paper we tried to identify all those gaps that is necessary for a trusted relationship between a service provider and service consumer. The aim of this research is to present an overview of the existing literature and compare them based on different criteria such as QoS integration, QoS repository, QoS filtering, trusted relationship and an SLA.
\end{abstract}

Keywords- Cloud Computing, Quality of Service (QOS); SLA; QOS Assessment, Trusted Relationship.

\section{INTRODUCTION}

Cloud services becoming a revolutionary addition to the computing era for every business to increase their efficiency and effectiveness. Computing, storage services are available whenever and wherever needed. As an adaptive system, cloud computing automatically balances loads and optimize the resource usage by sharing resources through multitenancy to many customers. Since the first adoption of cloud computing, many small and medium enterprises and individual users prefer cloud services to build their business systems. Increased popularity of cloud services is based on less cost and efficient resource usage [1].

In many domains, multiple cloud services providers have similar functional properties [2], such as in customer relationship management (CRM), different vendors offer variety of functionally equivalent services such as services offered by Microsoft Dynamic CRM, Salesforce Sales Cloud, SAP Sales OnDemand and Oracle Cloud CRM. It is very difficult for a consumer to select a suitable service provider manually. Security, privacy, integrity and Quality of Service (QOS) are critical factors for hindering the adoption and acceptance of cloud services. QOS is mainly non-functional properties of cloud service, which includes availability, reliability, responsiveness and security which is used as a main parameter for comparing between different service providers. On the other end a service provider need a reliable source for consumer previous resource usage record for optimally offer the amount of marginal resources to them [3]. Quality of Service (QOS) is assured by an SLA which is the main agreement between a consumer and the provider.

SLA is the documentation of promised expected performance, responsibilities and limits between service providers and users $[4,5]$. For forming a viable SLA, a service consumer need a centralized QoS repository and assessment framework that combine QoS information for offered services by the service provider from different sources and integrate them in decision making process. Similarly, the service provider particular small and medium cloud providers, need a reliable transaction history of requesting consumers to offer the accurate amount of marginal resources and to form a viable SLA with a consumer [6]. Cloud service trustworthiness is the total sum of client's perception of cloud service with respect to, reliability, scalability, availability, safety and security [2, 7]. For establishing a trusted relationship between a consumer and the provider, providers need to be customer focused and proactive in their marketing strategies to understand customer awareness of their services and a customer receives desired services in a promised level of expectation and in a cost effective way.

This paper attempts to integrate these findings by addressing following research question:

- How can a consumer and the provider be able to form a viable SLA in cloud environment by establishing and maintaining a trusted relationship using QoS repository and assessment framework? 
The rest of the paper is organized in such a way that, Section II presents classification of related existing approaches with their features, issues and working process. Section III discuss the comparative analysis of different approaches based on five parameters. Section IV discuss about findings and Section $\mathrm{V}$ concludes the paper with future research direction.

\section{LITERATURE REVIEW}

As discussed earlier, QoS is one of the primary factor in forming a viable SLA and for establishing a reliable relationship between two parties. There are substantial amount of literatures discussing about the topic [8-14]. We have divided these approaches into three classes based on their functionality and working attributes. The classes are:

- QoS in cloud service monitoring and prediction

- QoS in cloud SLA management

- QoS metrics in managing SLA

\section{A. QoS in Cloud Serivce Monitoring and Prediction}

One of the key issue business requirement is to assessing the quality of what service users are paying for in cloud environment. With the increasing complexity of components, middleware and infrastructure it is getting difficult to measure Key Performance Indicators (KPIs) of the service to be monitored. Authors [9] proposed a framework 'Quality of Service Monitoring as a Service (QoS-MONaaS)' that monitors QOS monitoring for an enterprise. The proposed framework increases the trust of the consumers by allowing them to get the full value of the service. To assess the cloud resources behavior to predict the service degradation authors [15] presented a Generalized Pareto Distribution models which are suitable for mobile clients and can track service degradation over time. These proposed approach evaluate short term extreme observations mainly bandwidth extremes and delays and proactively work to identify cloud services and infrastructure with degraded performance. Authors [16] proposed a framework that forecast and manage the cloud service based on QOS they have received at their end. Authors [11] proposed a Cross Layer Multi Cloud Application Monitoring and Benchmarking as a service for efficient QOS monitoring and benchmarking. The proposed framework monitors QOS of application components that may be deployed across multiple cloud platforms (Amazon EC2 and Microsoft Azure). In another study [12] authors introduced an approach which utilizes Social Network Analysis (SNA) principles along with Extreme Value Theorem (EVT) for a QOS prediction model. The proposed model depends on consumer's assessment of infrastructure service offerings. The proposed model delivers the decision of evaluation to the client. Service providers can collect data and then arrange suitable service offerings accordingly. Due to the multiplicity of cloud service providers, the perfect service provider who can satisfy consumer's requirements is very difficult to be decided. In many literatures the focal point is to determine the similarity between consumers and their services. But to introduce the challenge of personalized ranking prediction authors [17] predicted the rank on the basis of single QOS value. It can sometimes provide rank position for the same service based on different QOS parameters. Authors proposed six different kinds of correlated ranking algorithms and this approach improves the accuracy of the QOS ranking prediction. These QOS ranking predictions examine the order of services under consideration of a particular user. In our previous study $[18,19]$ we used consumer's previous profile history to assist the service provider to determine the amount of resources offer to consumer. Authors [20] recommended a cloud monitoring service approach which is flexible, autonomic, self-adjusting, platform-independent, reliable and useful for cloud providers and consumers. This proposed detects QOS violation and react to the violation with a proposed service substitution scheme. Service providers can visualize and manager performance at different levels by dashboard like indicators. However, the approach has different limitations in terms of proper service substitution and renegotiation. Authors [21] proposed a search based QoS prediction method. The approach measure similarity between two consumers by considering the occurrence probability of service pairs. Top-K neighbors were selected to provide QOS information for service ranking [8]. Authors [22] proposed a personalized ranking prediction framework - cloud rank, to predict QOS rankings without real-world service invocations. The previous service usage experiences of consumers help in personalized ranking prediction for other consumers. In another research authors [23] introduced a model for predicting end to end QOS values for cloud based services which is comprised of vertical composite service. Authors focused on QOS values and internal feature based similarity calculation to predict QoS values. For composite cloud services, authors [24] proposed a probabilistic modelling in which the QOS of an operation is drawn from a finite set of discrete values according to probability mass function. Authors [25] proposed a QoS prediction framework based on support vector regression algorithm, which solves the dimension issue of QOS assessment. The support vector regression algorithm has a good prediction effect on QOS of cloud manufacturing.

\section{B. QoS in Cloud SLA Management}

SLA is the keystone of the relationship between the service provider and a consumer. The main focus of any SLA is the specification and satisfaction of functional and nonfunctional requirements agreed between two parties. Both parties agreed to defined level of QoS for each services offered by the service provider and in term of violation define violation penalties. Similarly, the cloud provider, 
particularly small and medium provider is very cautious in forming a viable SLA and to allocate correct amount of marginal resources to consumers [13]. There are existing literatures discuss about QoS in SLA formation, which are described below.

Authors [14] used model driven framework to automatically generate SLA from the consumer requirements. The proposed method helps in SLA establishment guided by QoS parameters. The proposed three meta-models describe that how to combine a client functional and QoS requirements and assisting them in generating an optimal SLA. There are sets of rules to transform customer requirements into SLA derivation. In maximum cases, clients' functional and non-functional requirements are too complex to be in one relationship with a specific service provider. Integration of independent services can solve this problem. But in cloud service market a number of service providers are available who provide same function but with variable QoS for each services. To optimize the performance of composite service, the most appropriate service for each task should be selected. The QoS of this integrated service should match the constraints defined in SLA. To help cloud service providers to avoid SLA violation authors [26] proposed an approach that helps consumers to select the perfect composite service according to requirements and QOS constraints in SLA with the help of service selection algorithm. The QoS guarantee always explicit in SLA, which involves coordination and co-operation of many services and components their configuration and accurately their monitoring. Authors [27] proposed an integrated approach based on SLA enforcement for workflow application to enhance cloud capability and to guarantee QoS. So QoS guarantee from SLA perspective, involves co-ordination of many components and services, because these parameters are not static, it changes and the trust level of the service provider is not always as expected. Identifying that issue [28] introduced a novel concept of flexible SLA, to accommodate the fluctuated QoS parameters, based on which a trust evaluation method for cloud service provider can be started. The trust evaluation method is based on fluctuant QoS parameters and flexible SLA. Flexible and standard SLA preparation for diversified cloud services is a challenging task. Establishment of a clear SLA specific to a consumer and the provider is very crucial for achieving the objectives. Consumers and providers do not have same knowledge and may not share the same language. Identifying the necessity of understandable representation and automatic monitoring authors [29] introduced a formal readable and manageable SLA ontological model 'CSLAOnto' that is responsible for automatic detection of any violation and automatic monitoring of SLA. SLA management ensures that expected level of services should meet the criteria which are settled in their agreement and without real-time evaluation and adjustments of unexpected occurrence the management of SLA is ineffective. To address dynamic nature of cloud services authors [30] proposed an SLA monitoring framework that comprised of two sub-modules reputation assessment module and transactional risk assessment module. These two sub-modules assist a consumer in decision making for continuing using the same services or switching due to service level degradation. QoS values defined in SLAs but it may be different for different consumers depending on their requirements. Authors [31] proposed a framework 'COFILL, Collaborative Filtering with Location-based Data Smoothing' to focuses on data sparsity issues of QoS information and focused on historical QoS information by including consumers and service locations. The proposed approach work in two steps. In the first step the framework compute neighborhood of requesting consumers and service based on their location that offer a solid ground for data smoothing. In the second step both the user-based and service-based collaborative filtering method is used to predict QoS values. In another approach authors [32] proposed neighborhood based approach for collective and customized prediction of cloud components.

\section{QoS Metrics in Managing SLA}

For forming and managing a viable SLA it is very important to prepare QoS metrics before execution. These QoS metrics are a benchmark to evaluate the level of delivered and promised services and to assess that the services is up to the level or not. Diverse demands of clients are met up with different infrastructures such as IaaS, PasS or SaaS. However, the transformation of consumer's requirements into these infrastructures are a trivial job because the QoS for each services is not decided until the runtime. Therefore, it is a difficult job for a consumer to choose among service providers with similar products, distinct costs and various capability choices. For better assessment cloud services, the appropriate metrics should be prepared beforehand. Authors [33] proposed a QoS metrics to be assist the service providers in fulfilling consumer's priority. But this metrics is for provider only, because of diverse cloud services QoS metrics for evaluation for client has not been finalized yet. Though cloud service provider can evaluate the service level for the clients using proposed metrics, the level of service is dependent on SLA between two parties. But when providers go for external providers for extra resources, the promised service level is not assured anymore. In maximum cases, resource providers guarantee only availability, or other metrics which hinder efficient resource usage. Authors [34] proposed explorative statistic approach. The proposed approach used a new task scheduling metric that couple between tasks and resource optimal value. This metric assesses the scheduling under multiple QOS metrics and coupling between tasks. Authors [35] proposed a resource level metric for CPU performance 
guarantee in cloud SLA by resource level QoS metrics. The metric facilitates resource providers to dynamically allocate their resources among running services. Authors evaluate the framework by incorporating clients' CPU usage in metrics but avoiding fake SLA violations when client is not using all the allocated resources. In the workload allocation cases providers need to quickly plan and provision resources so that capacity infrastructure matches cloud workload. To have their workloads at minimal expense, consumer can design their application to maximize scaling. Authors [36] identified cloud workloads with their characteristics and constraints. The metrics based on QoS have been identified for each workload and have been analyzed for better application design. Authors [37] proposed a framework to dynamically monitor QOS metrics and performance measures to maintain SLA constraints. The proposed framework helps in detecting SLA violations and remedy action to take corrective measures. Authors [38] described SLA metrics for defining SLA for any layer of a cloud - IaaS, PaaS, SaaS. The SLA metrics is divided into two sections - performance metrics and business metrics. The performance metrics measure

Table 1: Critically analysis of existing approaches the performance parameters of data centres and the business metrics responsible for business related matters such as cost etc.

\section{CRITICAL ANALYSIS}

In this section, we have summarized and critically evaluate existing approaches. We discussed different approaches, their operational methods and objectives aiming QoS in forming SLAs. From existing literature, we tried to identify gaps and provide a basis for future research. We compared discussed existing approaches based on five criteria - QoS integration mechanism, QoS repository, QoS filtration, enabling interacting parties to establish and maintain trusted relationship and the use of QoS in SLAs. We believe that these parameters are very important for a consumer and the service provider for establishing and maintain trusted relationship by forming a viable SLA in cloud environment. The critical analysis of these approaches are presented in Table 1.

\begin{tabular}{|c|c|c|c|c|c|c|}
\hline Authors & Research Approach & $\begin{array}{c}\text { QOS } \\
\text { Integration }\end{array}$ & $\begin{array}{c}\text { QOS } \\
\text { Repository }\end{array}$ & $\begin{array}{l}\text { QOS } \\
\text { Filter }\end{array}$ & $\begin{array}{c}\text { Trusted } \\
\text { Relationship }\end{array}$ & $\begin{array}{c}\text { SLA } \\
\text { Perspective }\end{array}$ \\
\hline [9] & Quality of Service Monitoring as a Service & Yes & No & No & Yes & Yes \\
\hline [12] & Generalized Pareto Distribution model & No & No & No & Yes & Yes. \\
\hline [39] & $\begin{array}{c}\text { Multi-criteria decision } \\
\text { making method }\end{array}$ & No & Yes & No & Yes & No \\
\hline [16] & QOS monitoring for user devices & Yes & No & No & Yes & No \\
\hline [40] & mOSAIC based Intrusion Detection System & Yes & No & No & Yes & Yes \\
\hline [11] & $\begin{array}{l}\text { Cross-Layer Multi-Cloud Application Monitoring } \\
\text { and Benchmarking }\end{array}$ & No & No & No & Yes & No \\
\hline [20] & Platform independent approach & Yes & Yes & No & Yes & Yes \\
\hline$[14]$ & Model Driven Architecture & Yes & No & No & Yes & Yes \\
\hline$[26]$ & QOS Evaluation Method & No & No & No & Yes & Yes \\
\hline$[41]$ & Flexible SLA Model & No & Yes & No & Yes & Yes \\
\hline [29] & Ontology Based SLA & No & No & No & Yes & Yes \\
\hline$[30]$ & Real Time QOS Assessment & Yes & Yes & No & Yes & Yes \\
\hline$[2]$ & Trustworthiness Evaluation Framework & Yes & No & No & Yes & No \\
\hline$[33]$ & QOS Metrics for Service Providers & Yes & No & No & Yes & No \\
\hline$[35]$ & Resource Level Metric & Yes & No & No & Yes & Yes \\
\hline$[36]$ & Workload Based Metrics & Yes & No & No & No & No \\
\hline$[34]$ & Coupling Between Tasks Metric & Yes & No & No & No & No \\
\hline$[37]$ & Adaptive QOS Metrics Monitoring & Yes & No & No & Yes & Yes \\
\hline$[12]$ & SNA Based Algorithm & Yes & No & No & Yes & Yes \\
\hline$[17]$ & Correlated QOS Ranking Algorithm & Yes & No & No & Yes & No \\
\hline$[21]$ & Search based prediction approach & Yes & No & No & No & No \\
\hline$[22]$ & Cloud rank approach & Yes & No & No & No & No \\
\hline$[31]$ & $\begin{array}{c}\text { Collaborative Filtering with Location-based Data } \\
\text { Smoothing }\end{array}$ & No & No & No & No & No \\
\hline$[42]$ & CloudPred approach & Yes & No & No & No & No \\
\hline$[43]$ & Support Vector Approach & No & No & No & No & No \\
\hline$[23]$ & Vertical Composite Service & Yes & No & No & No & No \\
\hline$[24]$ & Probabilistic Modelling & No & No & No & No & No \\
\hline$[44]$ & Semantic Interoperability Approach & Yes & No & Yes & Yes & Yes \\
\hline$[45]$ & Semi-automated, Ontology Based Approach & Yes & Yes & No & Yes & Yes \\
\hline$[46]$ & Fuzzy User-oriented & Yes & No & No & No & No \\
\hline$[47]$ & Semantic Cloud Service Selection & Yes & No & No & No & No \\
\hline
\end{tabular}




\section{FINDINGS}

From thorough analysis of existing researches we found following gaps that need to be filled for establishing a reliable relationship between customer and the provider. The gaps are:

- Most of the approaches did not describe anything about merging QoS parameters from diversified sources for the decision making process and how to create a unified QoS repository for establishing a trusted relationship.

- There is no mechanism that align collected QoS from different sources into single uniform measurement scale.

- Most of the existing approaches do not differentiate between true and biased QoS information for decision making while forming an SLA. The QoS filtering is an important issue which cannot be ignored.

- Many of the existing approaches focus on offline trustworthiness evaluation, because offline trustworthiness evaluation provides more descriptive observations by past usage experience, however, online trustworthiness evaluation cannot be ignored which plays an important role in decision of service switching process.

- There is no mechanism that assist service requestor either a consumer or provider to choose a business context for identifying related QoS parameters.

- There is no mechanism that assist a service requestor to compare their required resources with the offered services based on multiple criteria and to offer a best match for required services and QoS parameter.

\section{CONCLUSION}

The application of cloud services is growing every next day, so the selection of suitable service provider, that fulfil business requirements and ensure quality of service for requested services are becoming more complex than ever before. The QoS is one of crucial factor in forming a viable SLA that assist both the service provider and a consumer. In this paper we have analyzed state of the art existing approaches that assist the interacting parties in forming a trusted relationship with each other. We compared these approaches based on five attributes which we believe are very important for the given problem. From critical analysis we identified certain gaps that need to be filled for forming a reliable and a viable SLA between the provider and a consumer. In our future work we will address all those gaps by proposing a framework to enable interacting parties to establish and maintain a trusted relationship with each other.

\section{REFERENCES}

2.

3. Hussain, W., et al., Provider-based optimized personalized viable SLA (OPV-SLA) framework to prevent SLA violation. The Computer Journal, 2016.

4. Hussain, W., F.K. Hussain, and O.K. Hussain. Maintaining Trust in Cloud Computing through SLA Monitoring. in Neural Information Processing. 2014. Springer.

5. Hussain, W., F.K. Hussain, and O.K. Hussain. SLA Management Framework to Avoid Violation in Cloud. in International Conference on Neural Information Processing. 2016. Springer.

6. Hussain, W., F.K. Hussain, and O. Hussain, QoS Prediction Methods to Avoid SLA Violation in Post-Interaction Time Phase, in 11th IEEE Conference on Industrial Electronics and Applications (ICIEA 2016) 2016, IEEE: Hefei, China.

7. Habib, S.M., S. Ries, and M. Muhlhauser. Towards a trust management system for cloud computing. in Trust, Security and Privacy in Computing and Communications (TrustCom), 2011 IEEE 10th International Conference on. 2011. IEEE.

8. Hussain, W., F.K. Hussain, and O. Hussain, Comparative analysis of consumer profile-based methods to predict SLA violation, in FUZZ-IEEE, IEEE, Editor. 2015, IEEE: Istanbul Turkey.

9. Adinolfi, O., et al. QoS-MONaaS: A Portable Architecture for QoS Monitoring in the Cloud. in 2012 Eighth International Conference on Signal Image Technology and Internet Based Systems. 2012.

10. Hussain, W., et al., Formulating and managing viable SLAs in cloud computing from a small to medium service provider's viewpoint: A state-of-the-art review. Information Systems, 2017. 71(Supplement C): p. 240-259.

11. Alhamazani, K., et al., Cross-layer multi-cloud real-time application QoS monitoring and benchmarking as-a-service framework. IEEE Transactions on Cloud Computing, 2015.

12. Kamel, A., A. Al-Fuqaha, and M. Guizani, Exploiting ClientSide Collected Measurements to Perform QoS Assessment of IaaS. IEEE Transactions on Mobile Computing, 2015. 14(9): p. $1876-1887$.

13. Hussain, W., F.K. Hussain, and O.K. Hussain. Towards Soft Computing Approaches for Formulating Viable Service Level Agreements in Cloud. in Neural Information Processing. 2015. Springer.

14. Boukadi, K., R. Grati, and H. Ben-Abdallah, Toward the automation of a QoS-driven SLA establishment in the Cloud. Service Oriented Computing and Applications, 2016. 10(3): p. 279-302.

15. Kamel, A., et al. Towards a client-side QoS monitoring and assessment using Generalized Pareto Distribution in a cloudbased environment. in 2013 IEEE Wireless Communications and Networking Conference Workshops (WCNCW). 2013.

16. ur Rehman, Z., et al., User-side QoS forecasting and management of cloud services. World Wide Web, 2015. 18(6): p. 1677-1716.

17. Jayapriya, K., N.A.B. Mary, and R.S. Rajesh, Cloud Service Recommendation Based on a Correlated QoS Ranking Prediction. Journal of Network and Systems Management, 2016. 24(4): p. 916-943

18. Hussain, W., et al., Profile-based viable Service Level Agreement (SLA) Violation Prediction Model in the Cloud, in 2015 10th International Conference on P2P, Parallel, Grid, 
Cloud and Internet Computing (3PGCIC). 2015, IEEE: Krakow, Poland. p. 268-272.

19. Hussain, W., F.K. Hussain, and O.K. Hussain. Comparative analysis of consumer profile-based methods to predict SLA violation. in Fuzzy Systems (FUZZ-IEEE), 2015 IEEE International Conference on. 2015. IEEE.

20. Serhani, M.A., Y. Atif, and A. Benharref, Towards an adaptive QoS-driven monitoring of cloud SaaS. International Journal of Grid and Utility Computing, 2014. 5(4): p. 263277.

21. Mao, C., et al., Search-based QoS ranking prediction for web services in cloud environments. Future Generation Computer Systems, 2015. 50: p. 111-126.

22. Zheng, Z., et al., QoS Ranking Prediction for Cloud Services. IEEE Transactions on Parallel and Distributed Systems, 2013. 24(6): p. 1213-1222.

23. Karim, R., C. Ding, and A. Miri. End-to-End QoS Prediction of Vertical Service Composition in the Cloud. in 2015 IEEE 8th International Conference on Cloud Computing. 2015.

24. Ngoko, Y., C. Cérin, and A. Goldman, Graph reduction for qos prediction of cloud-service compositions. International Journal of Business Process Integration and Management, 2014. 7(2): p. 89-102.

25. Liu, S., A Short-term Prediction for QoS of Cloud Manufacturing Based on Improved Support Vector Regression Algorithm. International Journal of u-and eService, Science and Technology, 2016. 9(6): p. 311-322.

26. Wang, D., et al., QoS and SLA Aware Web Service Composition in Cloud Environment. KSII Transactions on Internet \& Information Systems, 2016. 10(12).

27. Ait-Idir, M. and N. Agoulmine. Enhancing cloud capabilities for SLA enforcement of cloud scheduled applications. in Proceedings of the 9th International Conference on Utility and Cloud Computing. 2016. ACM.

28. Qi, L., et al. A Trust Evaluation Method for Cloud Service with Fluctuant QoS and Flexible SLA. in 2014 IEEE International Conference on Web Services. 2014.

29. Labidi, T., A. Mtibaa, and H. Brabra, CSLAOnto: A Comprehensive Ontological SLA Model in Cloud Computing. Journal on Data Semantics, 2016. 5(3): p. 179-193.

30. Hammadi, A.M. and O. Hussain. A Framework for SLA Assurance in Cloud Computing. in 2012 26th International Conference on Advanced Information Networking and Applications Workshops. 2012.

31. Tang, M., et al., Cloud service QoS prediction via exploiting collaborative filtering and location-based data smoothing. Concurrency and Computation: Practice and Experience, 2015. 27(18): p. 5826-5839.

32. Zhang, Y., Z. Zheng, and M.R. Lyu. Exploring latent features for memory-based QoS prediction in cloud computing. in Reliable Distributed Systems (SRDS), 2011 30th IEEE Symposium on. 2011. IEEE.

33. Bardsiri, A.K. and S.M. Hashemi, Qos metrics for cloud computing services evaluation. International Journal of Intelligent Systems and Applications, 2014. 6(12): p. 27.
34. Mohan, V.M. and K. Satyanarayana, Efficient task scheduling strategy towards QoS aware optimal resource utilization in cloud computing. Journal of Theoretical and Applied Information Technology, 2015. 80(1): p. 152.

35. Goiri, Í., et al., Supporting CPU-based guarantees in cloud SLAs via resource-level QoS metrics. Future Generation Computer Systems, 2012. 28(8): p. 1295-1302.

36. Singh, S. and I. Chana, Metrics based Workload Analysis Technique for IaaS Cloud. CoRR, 2014. abs/1411.6753.

37. Khan, H.M., G.Y. Chan, and F.F. Chua. An adaptive monitoring framework for ensuring accountability and quality of services in cloud computing. in 2016 International Conference on Information Networking (ICOIN). 2016.

38. Alhamad, M., T. Dillon, and E. Chang. Conceptual SLA framework for cloud computing. in Digital Ecosystems and Technologies (DEST), 2010 4th IEEE International Conference on. 2010. IEEE.

39. Radovanović, S., et al. Cloud-based framework for $Q o S$ monitoring and provisioning in consumer devices. in 2013 IEEE Third International Conference on Consumer Electronics i\& Berlin (ICCE-Berlin). 2013.

40. Ficco, M., M. Rak, and B. Di Martino. An intrusion detection framework for supporting sla assessment in cloud computing. in Computational Aspects of Social Networks (CASoN), 2012 Fourth International Conference on. 2012. IEEE.

41. Qi, L., et al. A trust evaluation method for cloud service with fluctuant qos and flexible sla. in Web Services (ICWS), 2014 IEEE International Conference on. 2014. IEEE.

42. Zhang, Y., Z. Zheng, and M.R. Lyu. Exploring Latent Features for Memory-Based QoS Prediction in Cloud Computing. in 2011 IEEE 30th International Symposium on Reliable Distributed Systems. 2011.

43. Liu, S., A Short-term Prediction for QoS of Cloud Manufacturing Based on Improved Support Vector Regression Algorithm. International Journal of u-and eService, Science and Technology, 2016. 9: p. 311-322.

44. Fortis, T.F., V.I. Munteanu, and V. Negru. Towards an Ontology for Cloud Services. in 2012 Sixth International Conference on Complex, Intelligent, and Software Intensive Systems. 2012

45. Zhang, M., et al. An ontology-based system for Cloud infrastructure services' discovery. in 8th International Conference on Collaborative Computing: Networking, Applications and Worksharing (CollaborateCom). 2012.

46. Sun, L., et al., Cloud-FuSeR: Fuzzy ontology and MCDM based cloud service selection. Future Generation Computer Systems, 2016. 57: p. 42-55.

47. Junhao, W., et al. Semantic Web Service Selection Algorithm Based on QoS Ontology. in 2011 International Joint Conference on Service Sciences. 2011. 